Supporting Information

\title{
One-step Synthesis of Sugar Nucleotides
}

Atsushi Miyagawa ${ }^{*}, \dagger$, , Sanami Toyama ${ }^{\dagger}$, Ippei Ohmura ${ }^{\ddagger}$, Shun Miyazaki ${ }^{\ddagger}$, Takeru Kamiya ${ }^{\ddagger}$, Hatsuo Yamamura ${ }^{\dagger, \dagger}$

†Department of Materials Science and Engineering, Nagoya Institute of Technology, Gokiso, Showa-ku, Nagoya, 466-8555, Japan

‡Department of Life Science and Applied Chemistry, Nagoya Institute of Technology, Gokiso, Showa-ku, Nagoya, 466-8555, Japan

Corresponding author. E-mail: miyagawa.atsushi@ nitech.ac.jp; Tel.: +81-52 (735) 5239

Contents

1) ${ }^{1} \mathrm{H},{ }^{13} \mathrm{C}$ and ${ }^{31} \mathrm{P}$ NMR, ${ }^{1} \mathrm{H}^{-1} \mathrm{H}$ COSY, ${ }^{1} \mathrm{H}^{13}{ }^{13} \mathrm{C}$ HMQC and ESI-MS spectra of UDP-glucose 1.....S2

2) ${ }^{1} \mathrm{H},{ }^{13} \mathrm{C}$ and ${ }^{31} \mathrm{P}$ NMR,${ }^{1} \mathrm{H}-{ }^{1} \mathrm{H}$ COSY,${ }^{1} \mathrm{H}-{ }^{13} \mathrm{C}$ HMQC and ESI-MS spectra of UMP-glucose 2 .....S5

3) ${ }^{1} \mathrm{H},{ }^{13} \mathrm{C}$ and ${ }^{31} \mathrm{P}$ NMR,,${ }^{1} \mathrm{H}-{ }^{1} \mathrm{H}$ COSY,${ }^{1} \mathrm{H}_{-}{ }^{13} \mathrm{C}$ HMQC and ESI-MS spectra of UDP-galactose $3 \ldots \mathrm{S} 8$

4) ${ }^{1} \mathrm{H},{ }^{13} \mathrm{C}$ and ${ }^{31} \mathrm{P}$ NMR, ${ }^{1} \mathrm{H}-{ }^{1} \mathrm{H} \mathrm{COSY},{ }^{1} \mathrm{H}^{1}{ }^{13} \mathrm{C}$ HMQC and ESI-MS spectra of UDP-mannose 4...S11

5) ${ }^{1} \mathrm{H},{ }^{13} \mathrm{C}$ and ${ }^{31} \mathrm{P}$ NMR,,${ }^{1} \mathrm{H}-{ }^{1} \mathrm{H}$ COSY, ${ }^{1} \mathrm{H}^{-1}{ }^{13} \mathrm{C}$ HMQC and ESI-MS spectra of GDP-mannose 5...S 14 
1) ${ }^{1} \mathrm{H},{ }^{13} \mathrm{C}$ and ${ }^{31} \mathrm{P}$ NMR, ${ }^{1} \mathrm{H}-{ }^{1} \mathrm{H}$ COSY,${ }^{1} \mathrm{H}-{ }^{13} \mathrm{C}$ HMQC and ESI-MS spectra of UDP-glucose $\mathbf{1}$ ${ }^{1} \mathrm{H}$ NMR spectrum of UDP-glucose $1\left(400 \mathrm{MHz}, \mathrm{D}_{2} \mathrm{O}\right)$

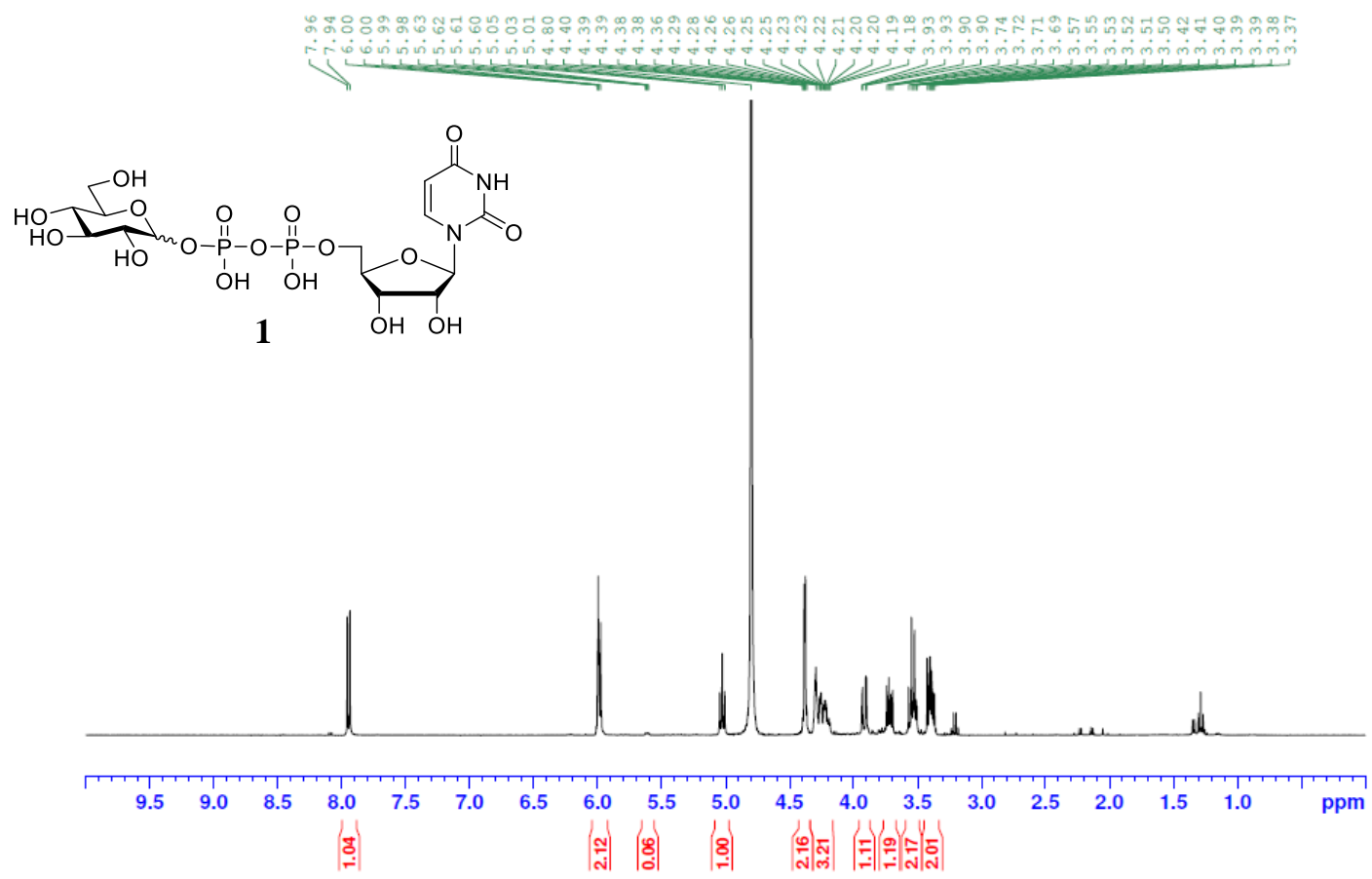

${ }^{13} \mathrm{C}$ NMR spectrum of UDP-glucose $1\left(101 \mathrm{MHz}, \mathrm{D}_{2} \mathrm{O}\right)$

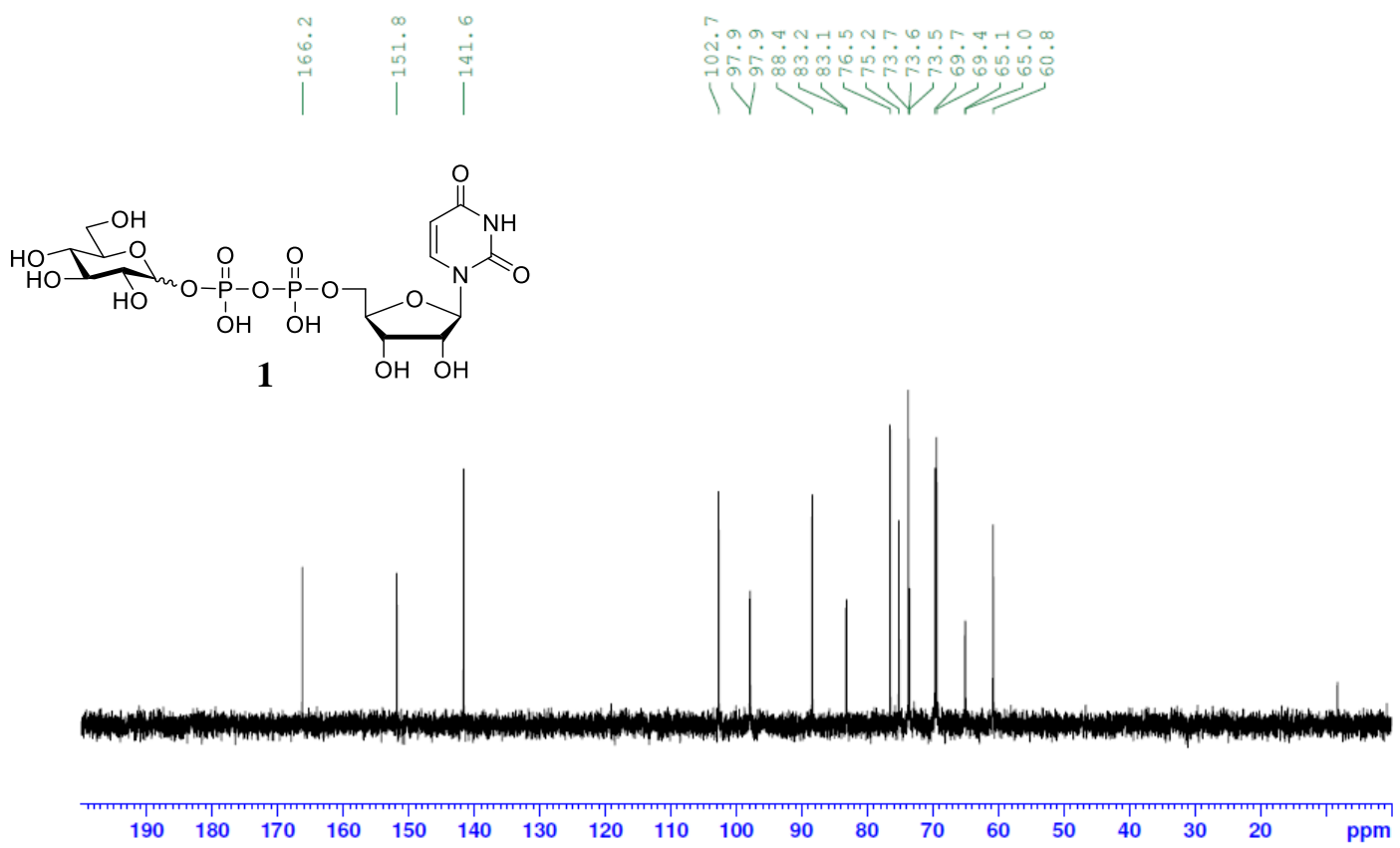


${ }^{31} \mathrm{P}$ NMR spectrum of UDP-glucose $1\left(162 \mathrm{MHz}, \mathrm{D}_{2} \mathrm{O}\right)$
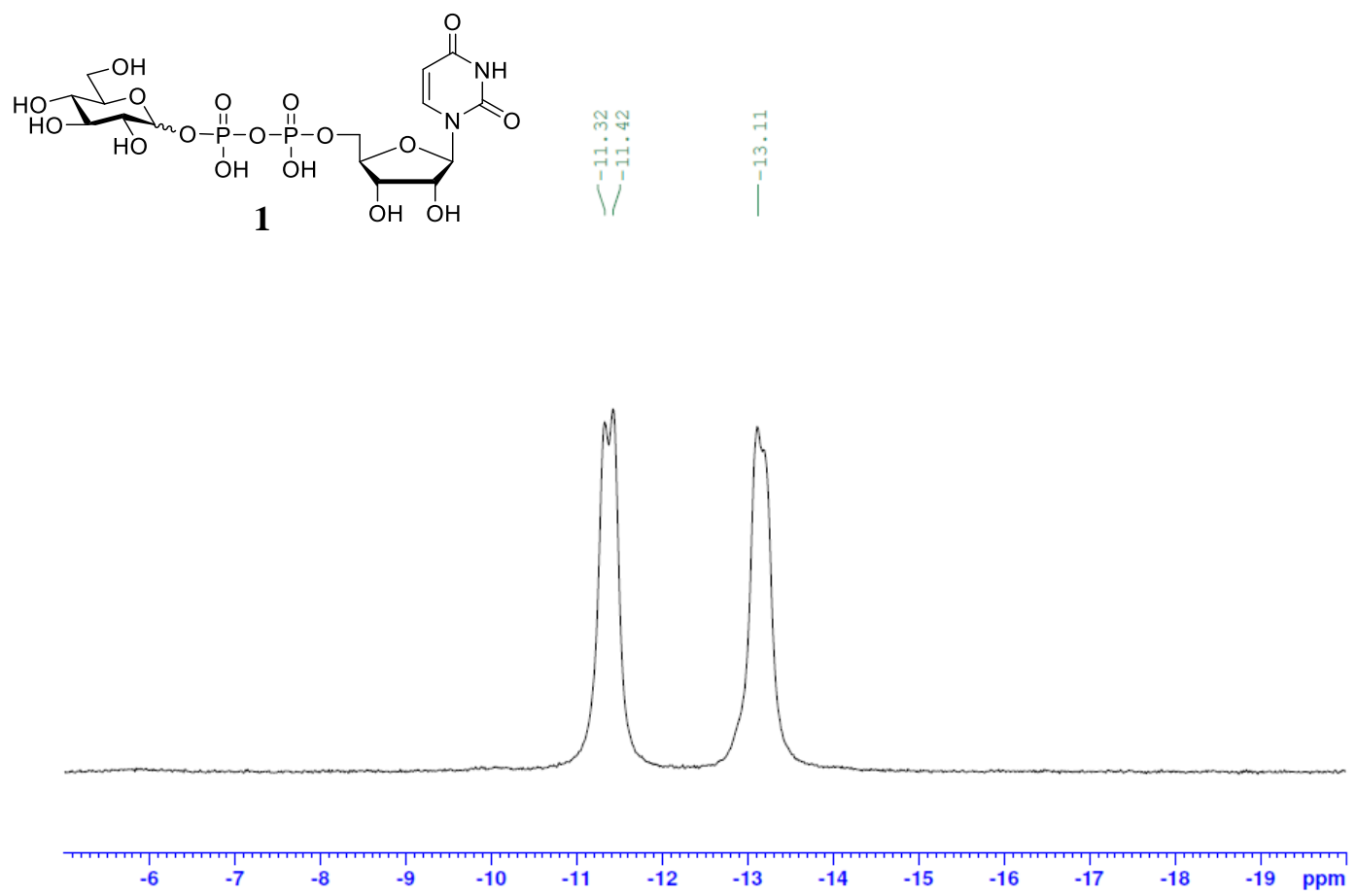

${ }^{1} \mathrm{H}-{ }^{1} \mathrm{H}$ COSY of UDP-glucose $\mathbf{1}\left(\mathrm{D}_{2} \mathrm{O}\right)$

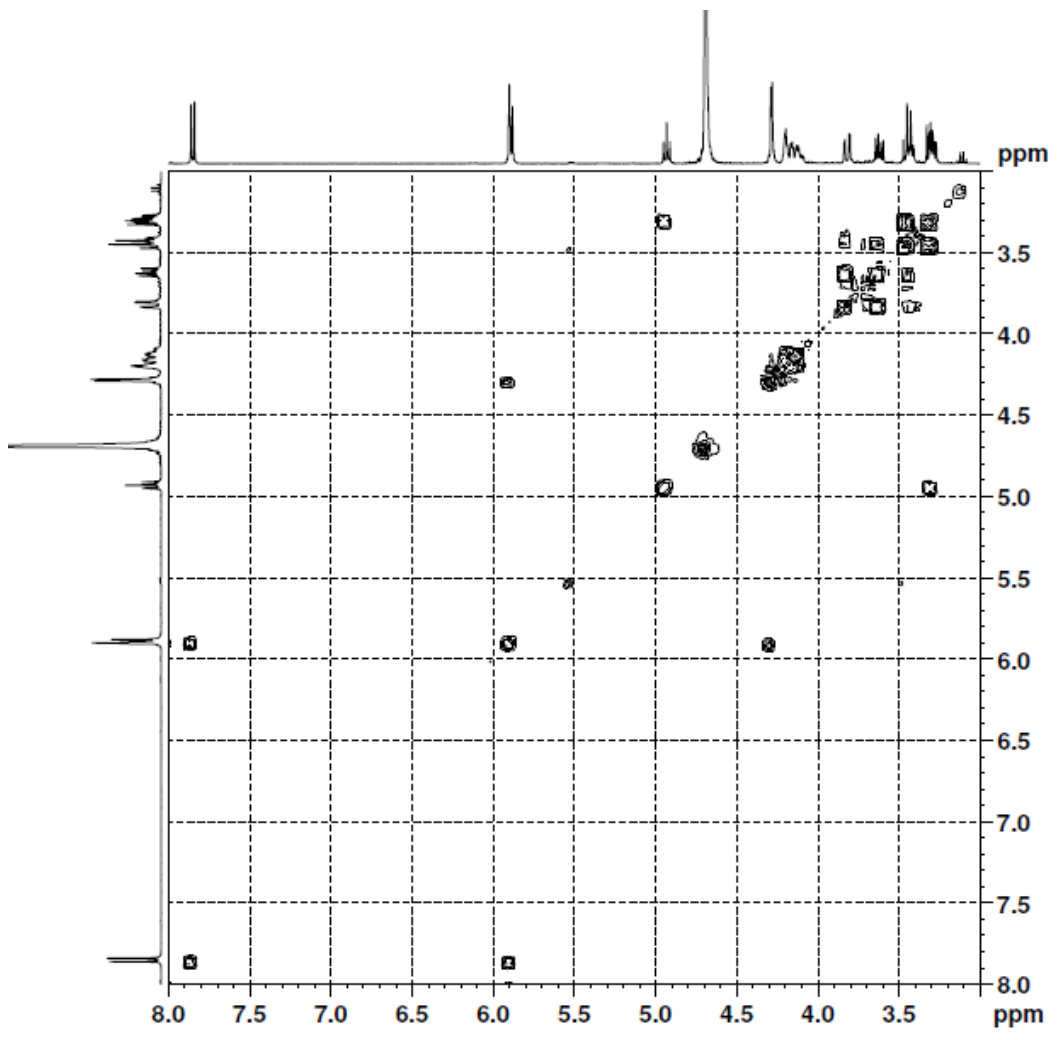


${ }^{1} \mathrm{H}-{ }^{13} \mathrm{C}$ HMQC of UDP-glucose $\mathbf{1}\left(\mathrm{D}_{2} \mathrm{O}\right)$

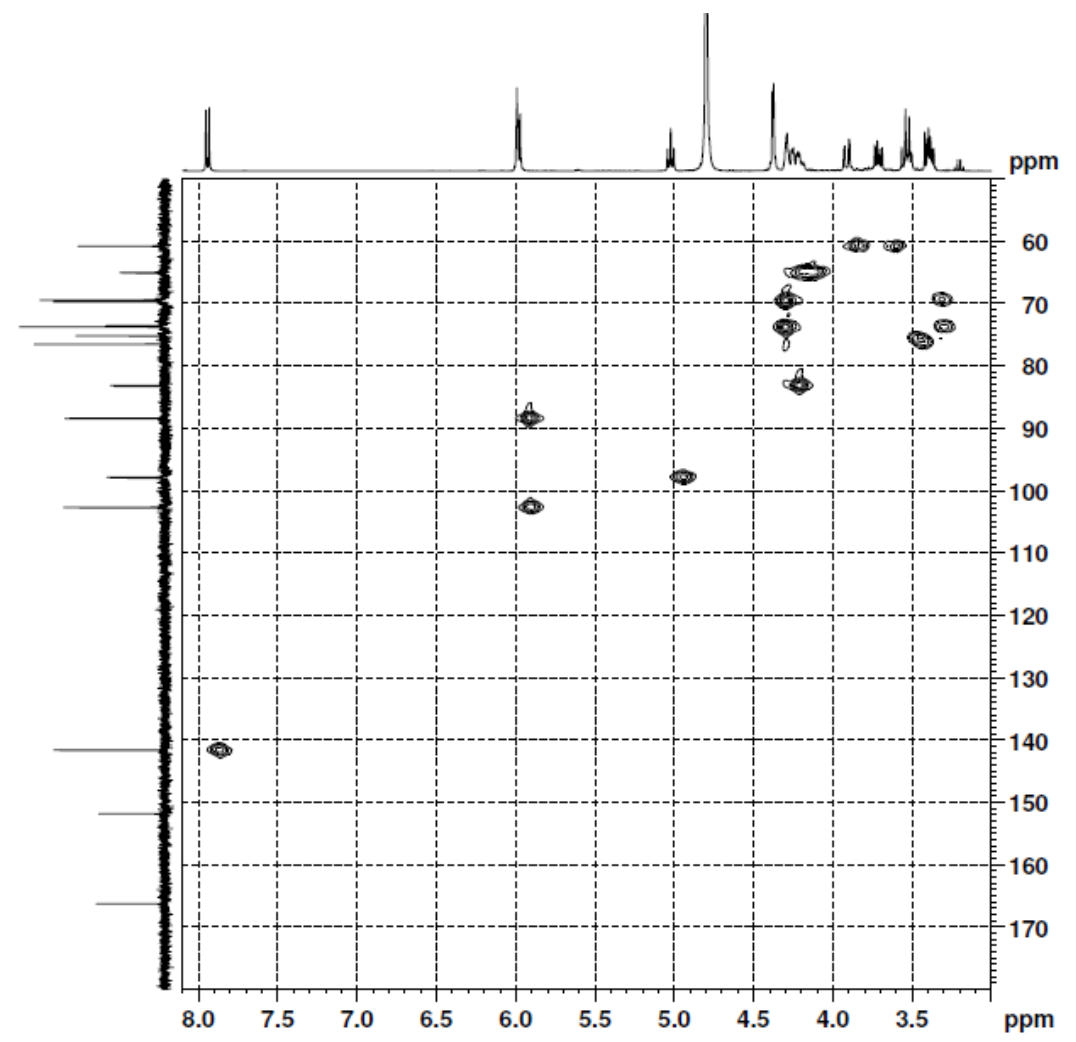

ESI-HRMS spectrum of UDP-glucose 1

160906_001 25 (0.312) AM2 (Ar, 18000.0,0.00,0.00); Cm (25:30-121:131) 100

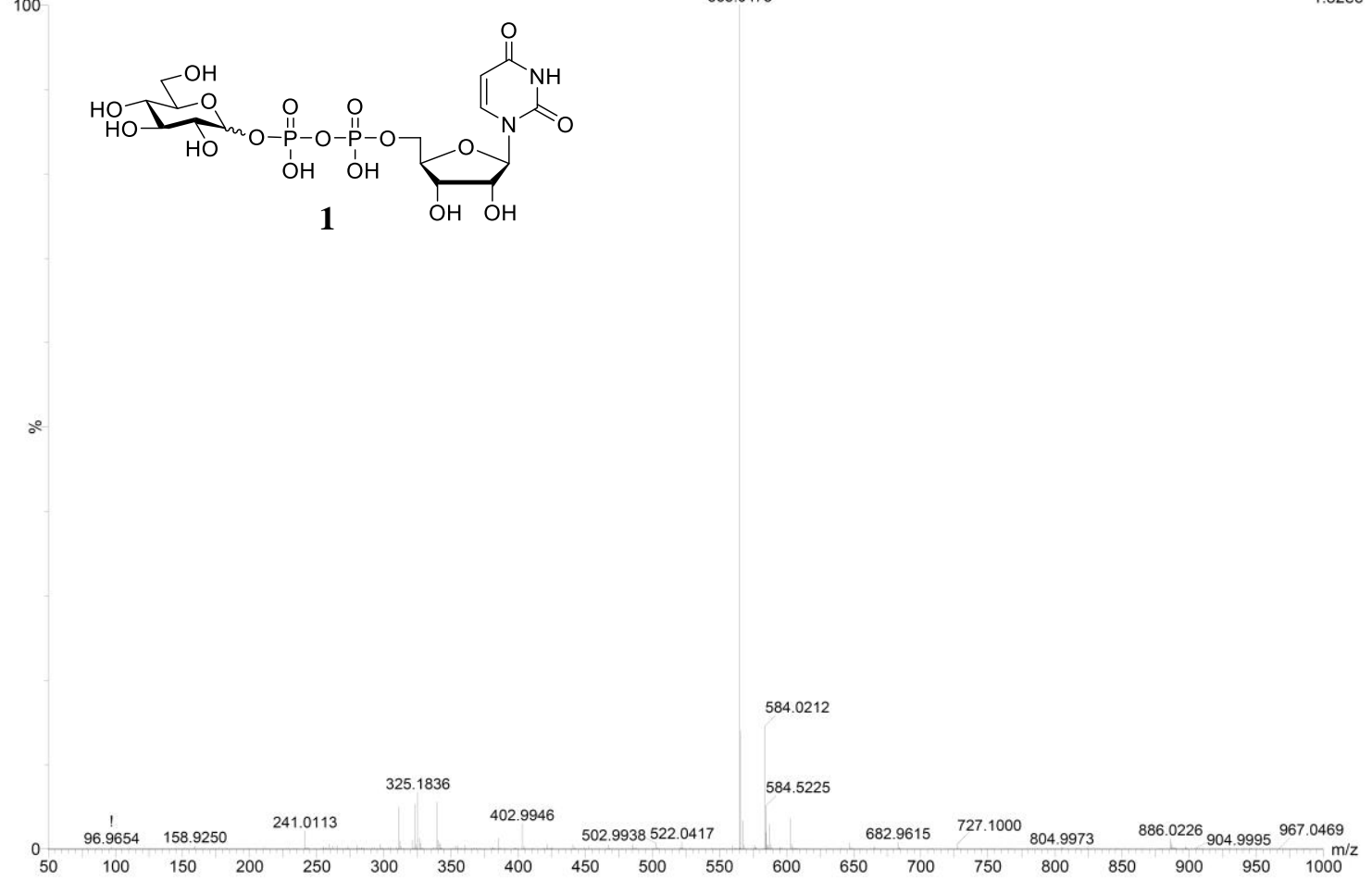

565.0475

1: TOF MS ES$1.82 \mathrm{e} 6$ 
2) ${ }^{1} \mathrm{H},{ }^{13} \mathrm{C}$ and ${ }^{31} \mathrm{P}$ NMR, ${ }^{1} \mathrm{H}-{ }^{-1} \mathrm{H}$ COSY,${ }^{1} \mathrm{H}^{-13} \mathrm{C}$ HMQC and ESI-MS spectra of UMP-glucose 2 ${ }^{1} \mathrm{H}$ NMR spectrum of UMP-glucose $2\left(400 \mathrm{MHz}, \mathrm{D}_{2} \mathrm{O}\right)$

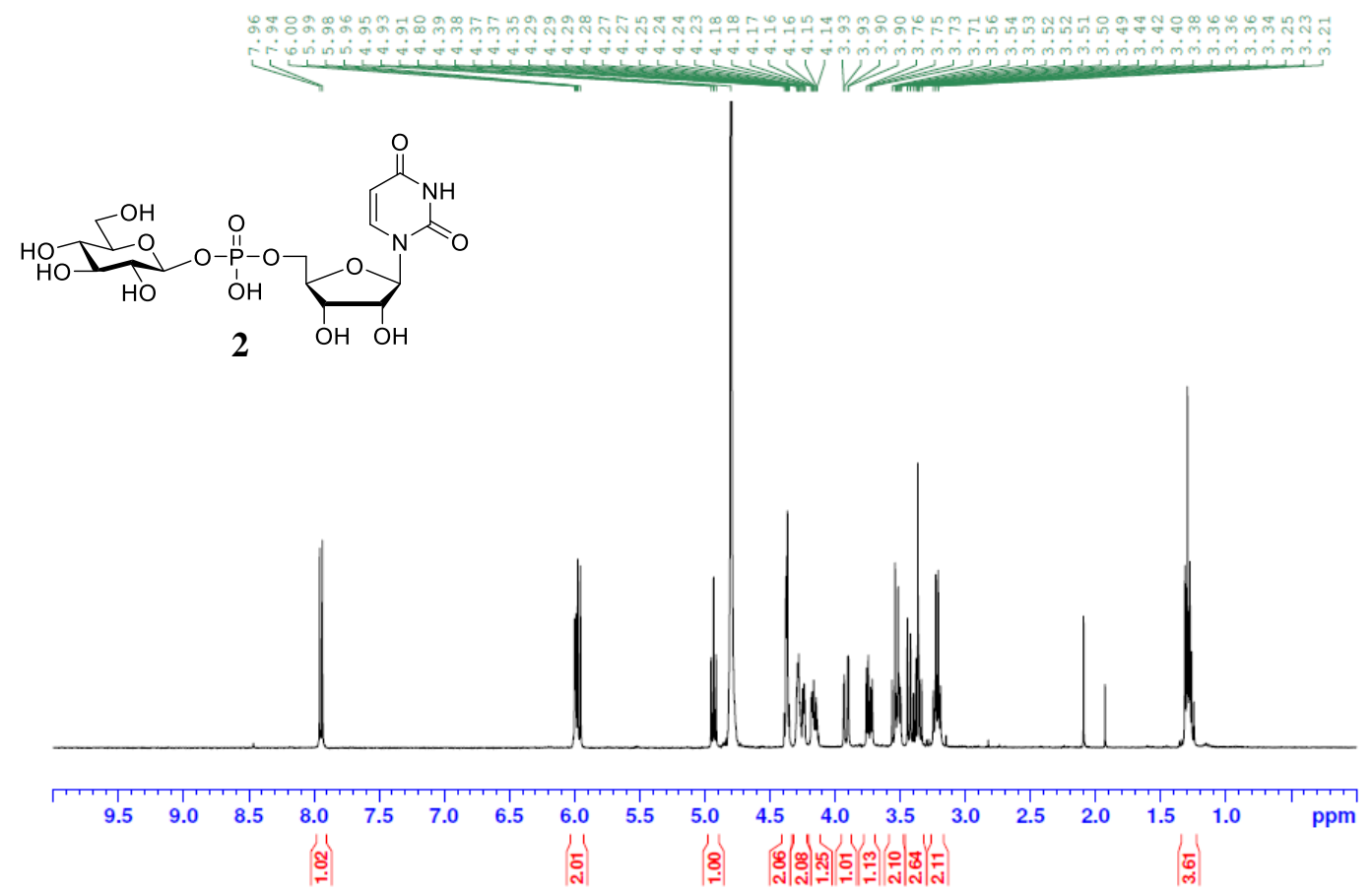

${ }^{13} \mathrm{C}$ NMR spectrum of UMP-glucose $2\left(101 \mathrm{MHz}, \mathrm{D}_{2} \mathrm{O}\right)$

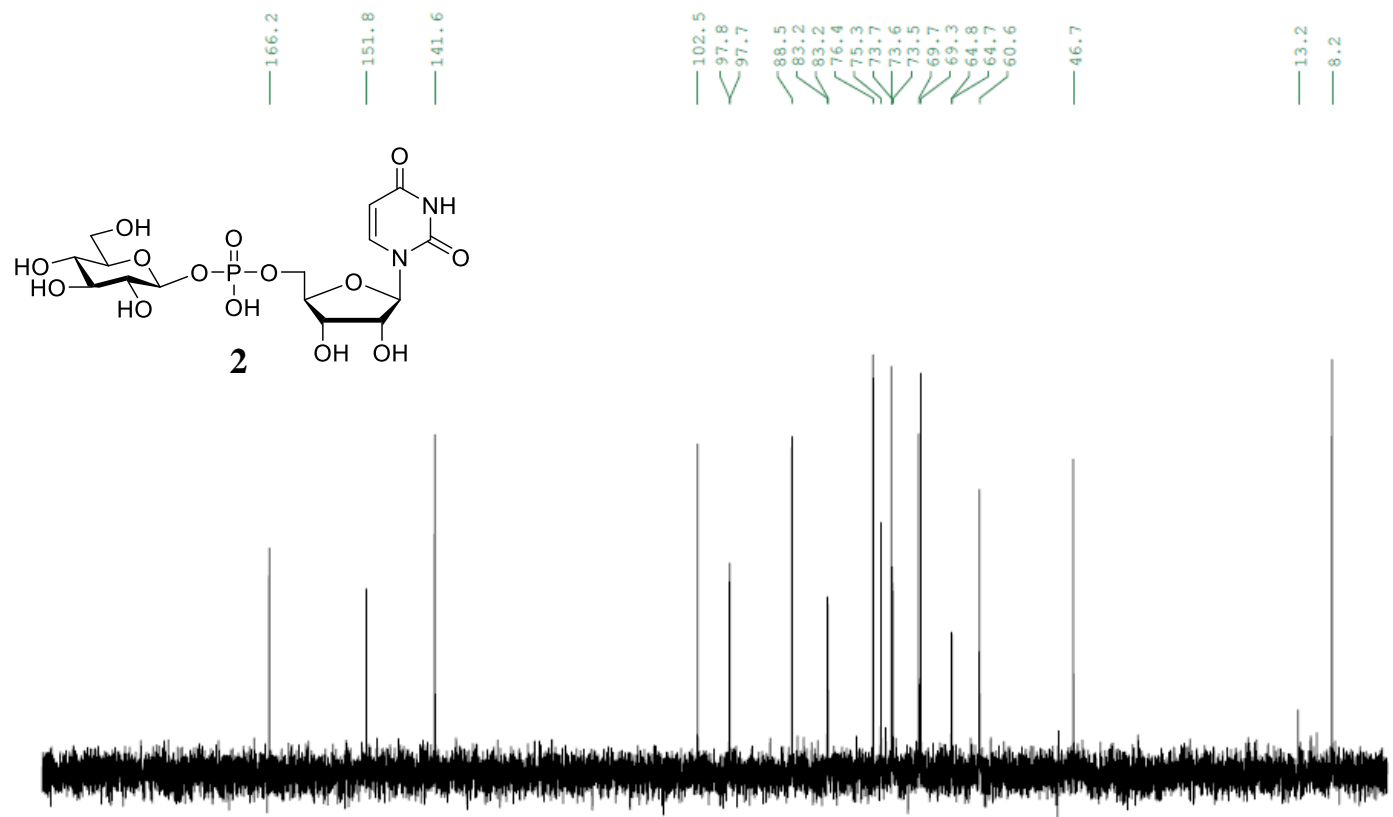

$\begin{array}{lllllllllllllllllll}190 & 180 & 170 & 160 & 150 & 140 & 130 & 120 & 110 & 100 & 90 & 80 & 70 & 60 & 50 & 40 & 30 & 20 & \mathrm{ppm}\end{array}$ 
${ }^{31} \mathrm{P}$ NMR spectrum of UMP-glucose $2\left(162 \mathrm{MHz}, \mathrm{D}_{2} \mathrm{O}\right)$
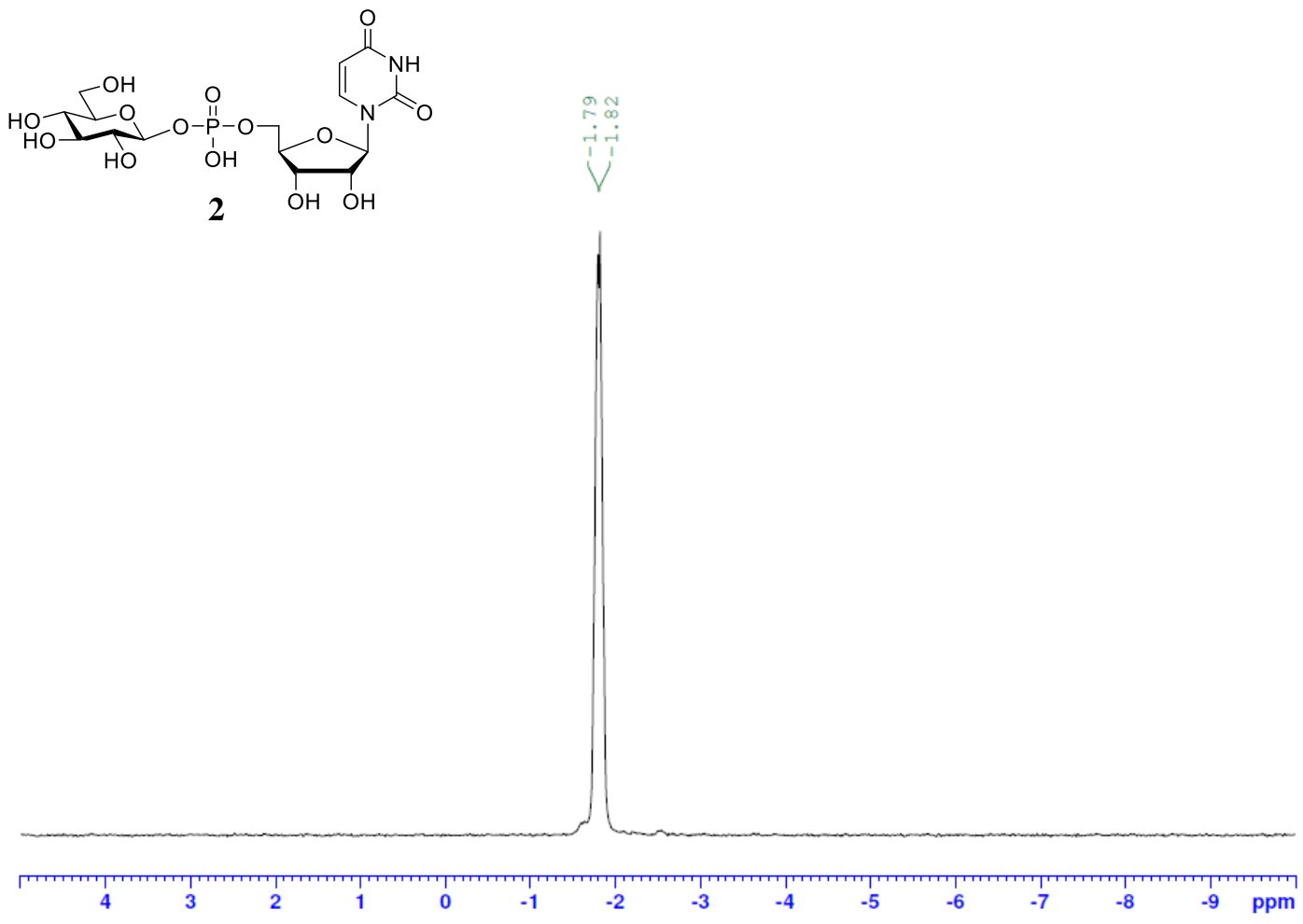

${ }^{1} \mathrm{H}-{ }^{1} \mathrm{H}$ COSY of UMP-glucose $2\left(\mathrm{D}_{2} \mathrm{O}\right)$

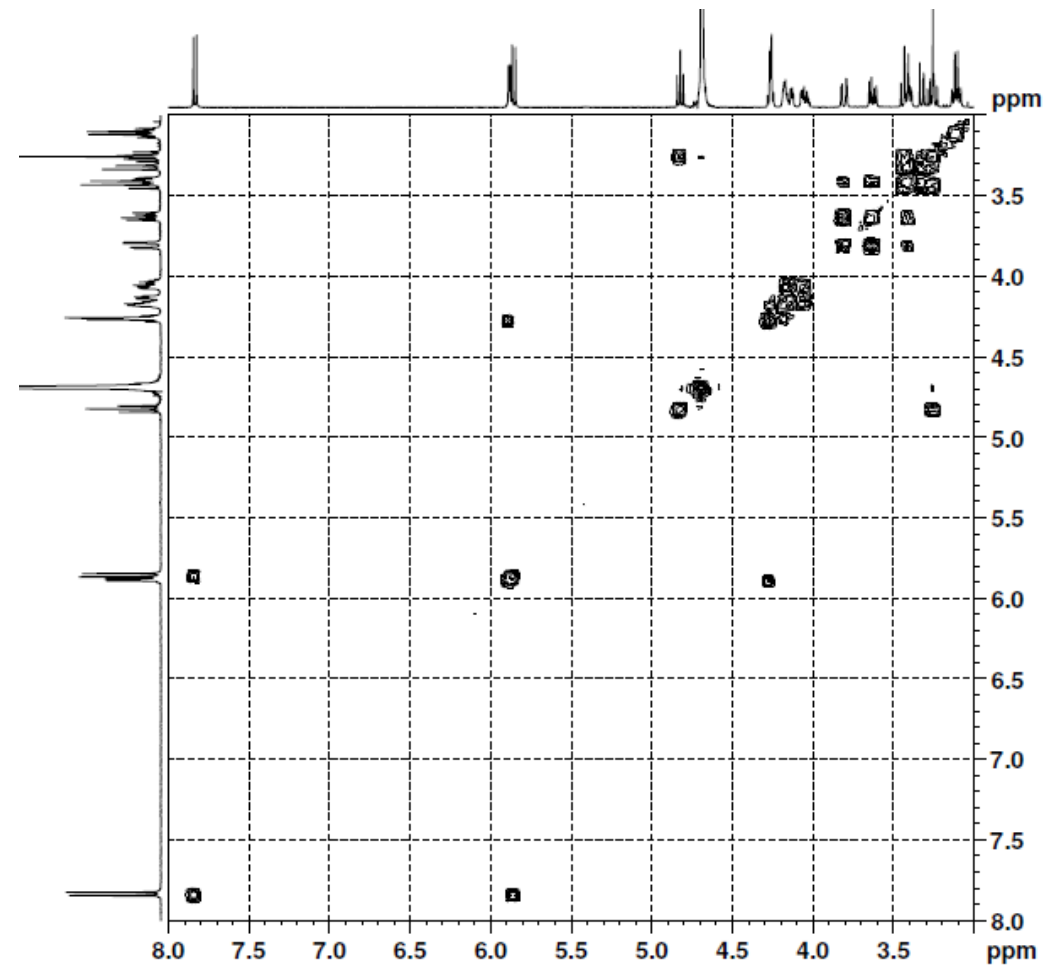


${ }^{1} \mathrm{H}-{ }^{13} \mathrm{C}$ HMQC of UMP-glucose $2\left(\mathrm{D}_{2} \mathrm{O}\right)$

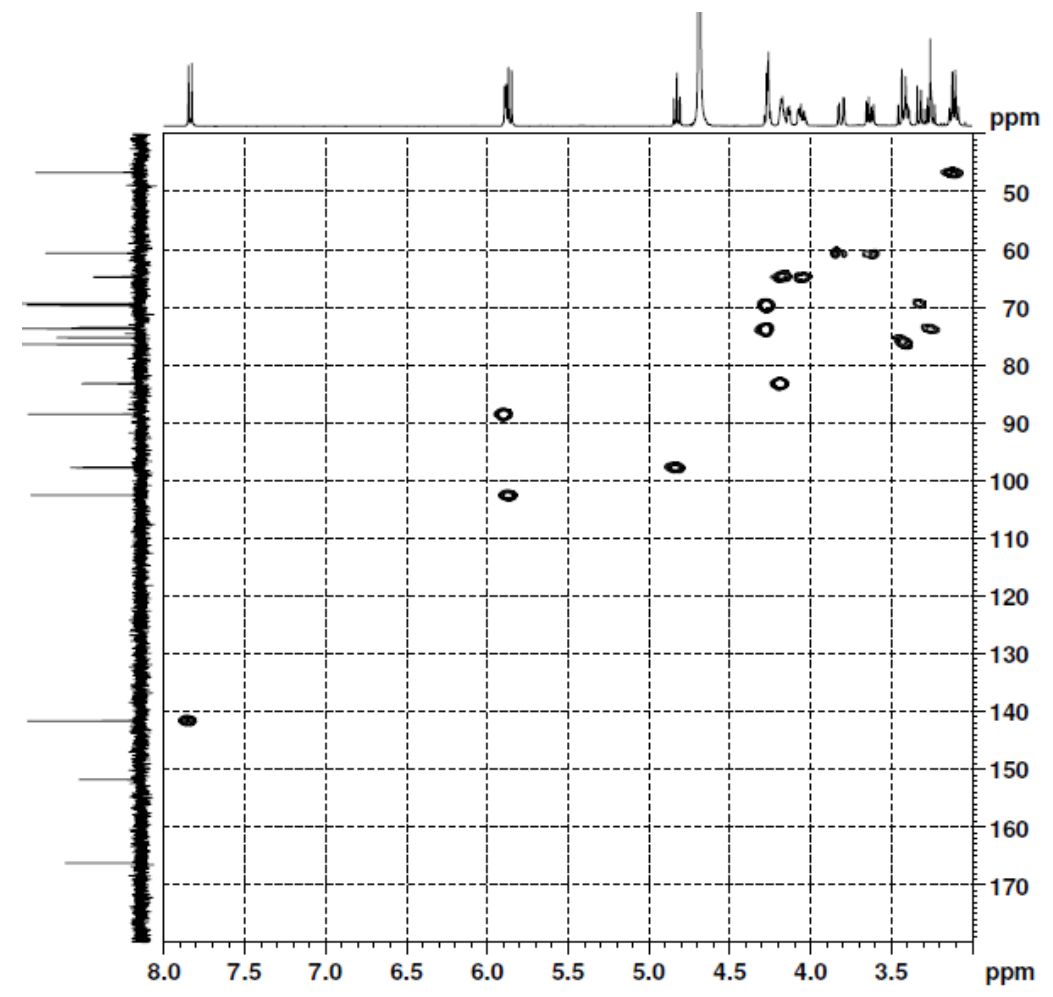

ESI-HRMS spectrum of UMP-glucose 2

160906_002 25 (0.312) AM2 (Ar, 18000.0,0.00,0.00); Cm (24:29-112:122) 100

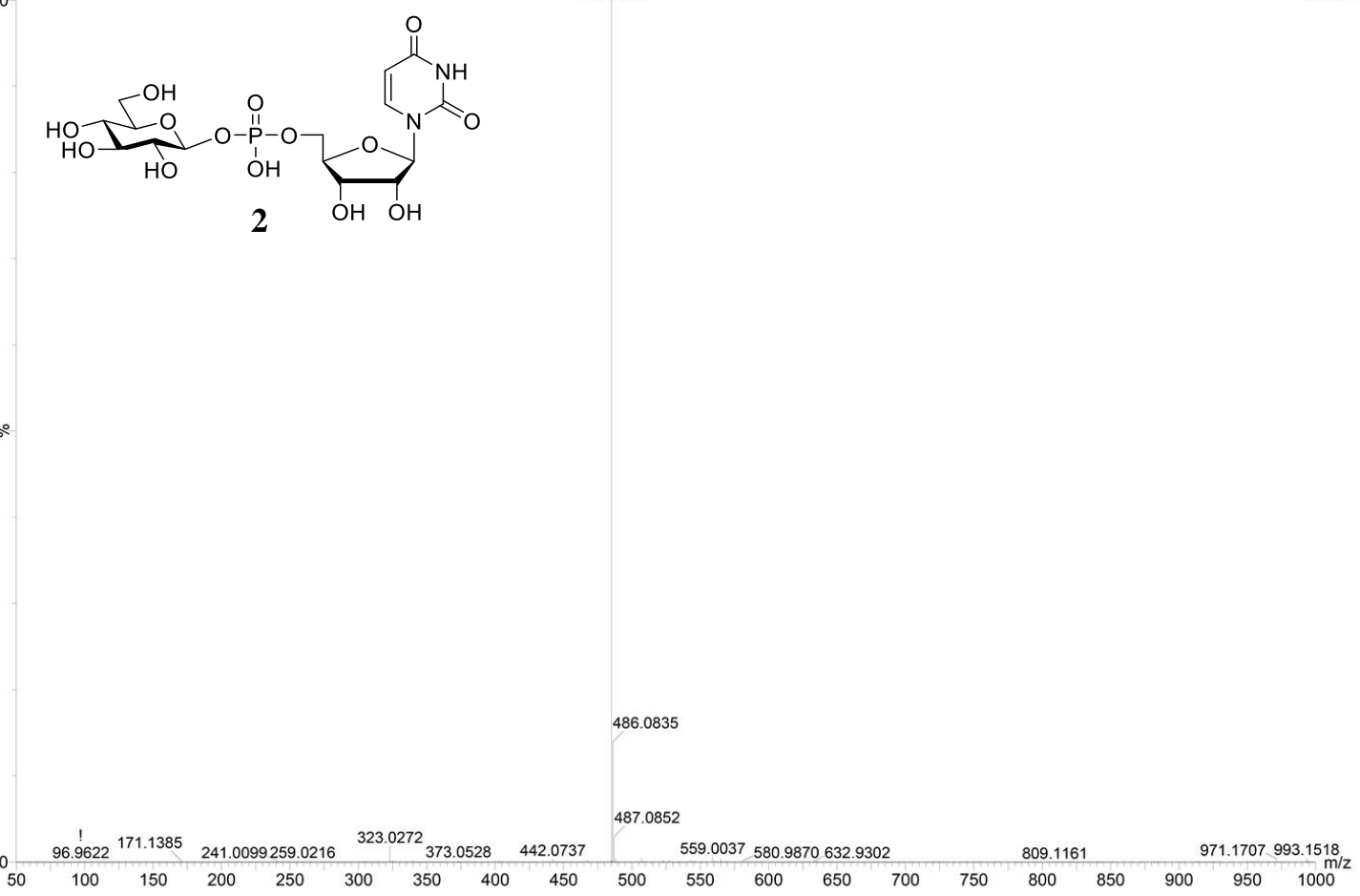


3) ${ }^{1} \mathrm{H},{ }^{13} \mathrm{C}$ and ${ }^{31} \mathrm{P}$ NMR,,${ }^{1} \mathrm{H}-{ }^{1} \mathrm{H}$ COSY,${ }^{1} \mathrm{H}-{ }^{13} \mathrm{C}$ HMQC and ESI-MS spectra of UDP-galactose 3 ( $\alpha: \beta$ $=1: 13$ )

${ }^{1} \mathrm{H}$ NMR spectrum of UDP-galactose $3\left(400 \mathrm{MHz}, \mathrm{D}_{2} \mathrm{O}\right)$

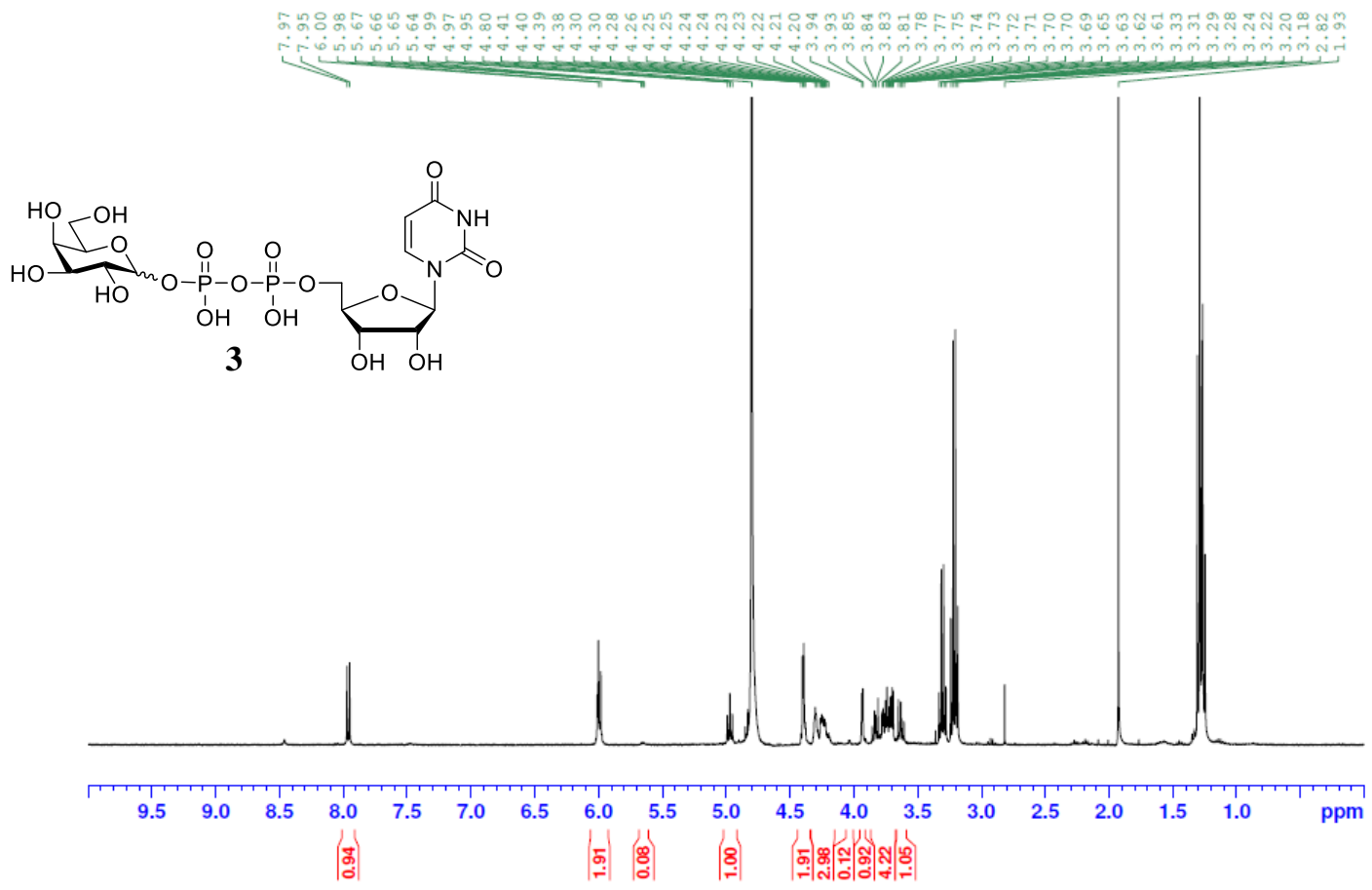

${ }^{13} \mathrm{C}$ NMR spectrum of UDP-galactose $3\left(101 \mathrm{MHz}, \mathrm{D}_{2} \mathrm{O}\right)$<smiles>O=CC1CCCCCC1C=O</smiles>
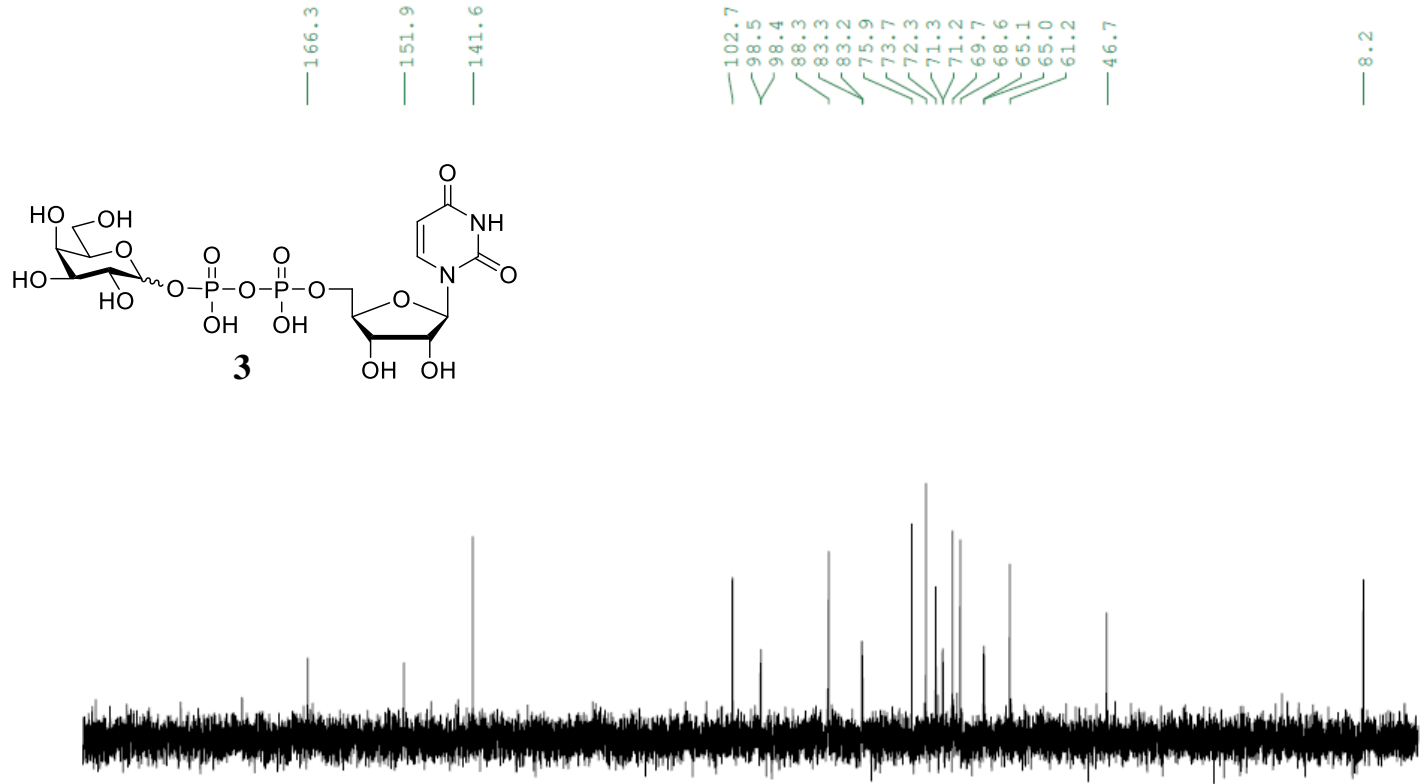

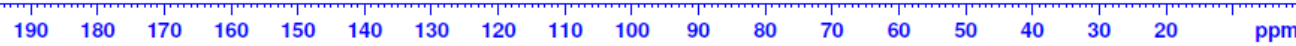


${ }^{31} \mathrm{P}$ NMR spectrum of UDP-galactose $3\left(162 \mathrm{MHz}, \mathrm{D}_{2} \mathrm{O}\right)$

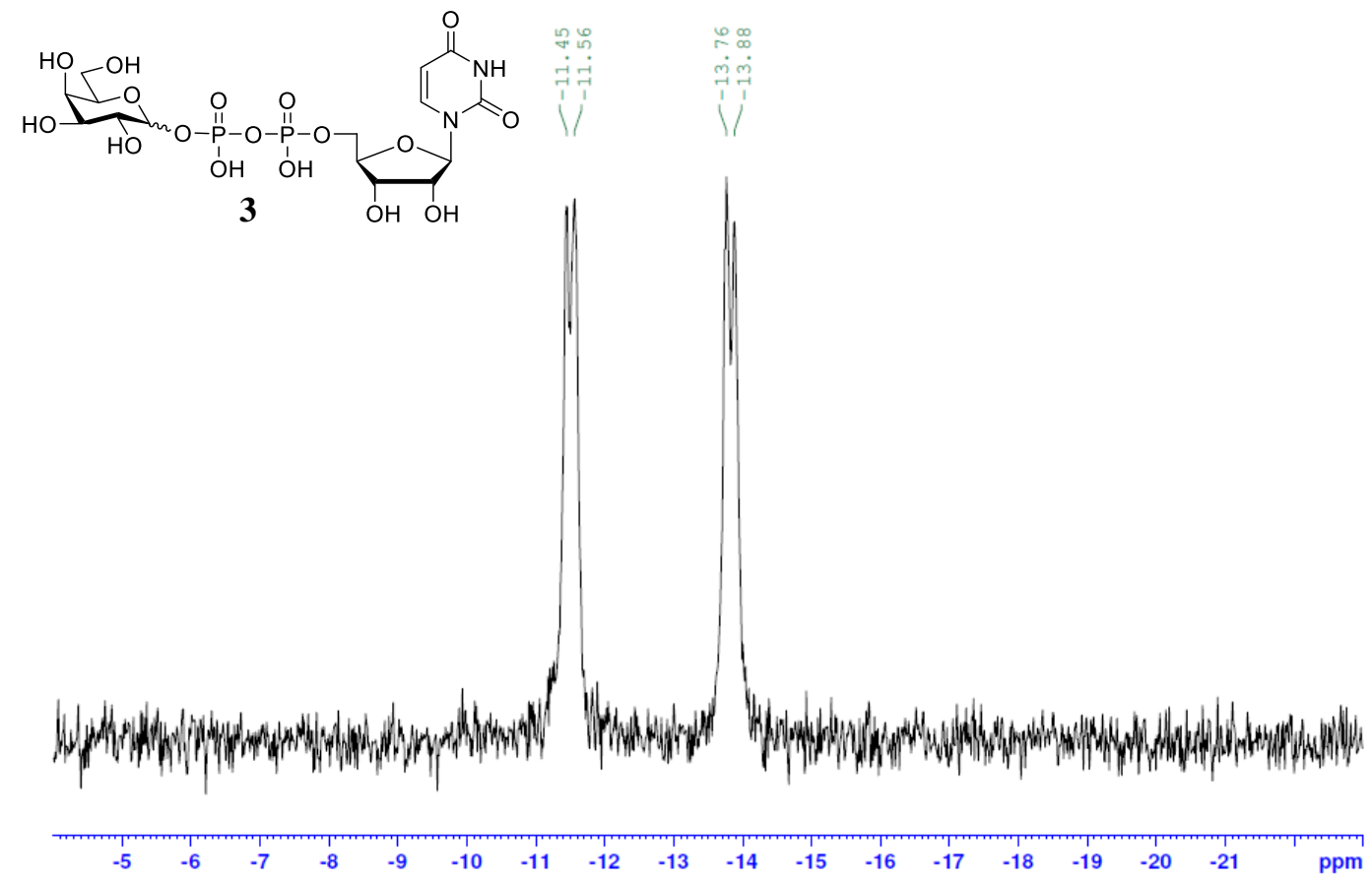

${ }^{1} \mathrm{H}-{ }^{1} \mathrm{H}$ COSY of UDP-galactose $3\left(\mathrm{D}_{2} \mathrm{O}\right)$

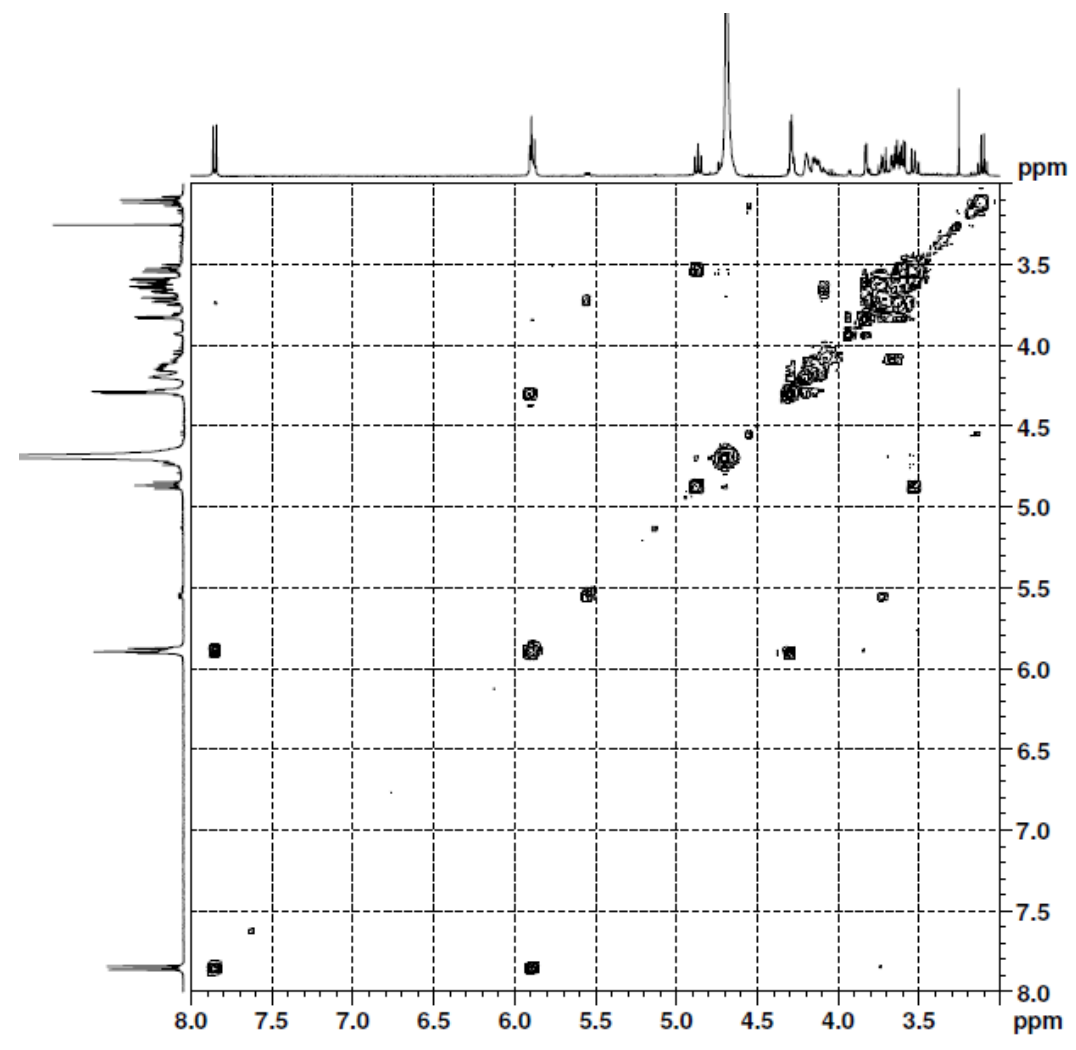


${ }^{1} \mathrm{H}-{ }^{13} \mathrm{C}$ HMQC of UDP-galactose $3\left(\mathrm{D}_{2} \mathrm{O}\right)$

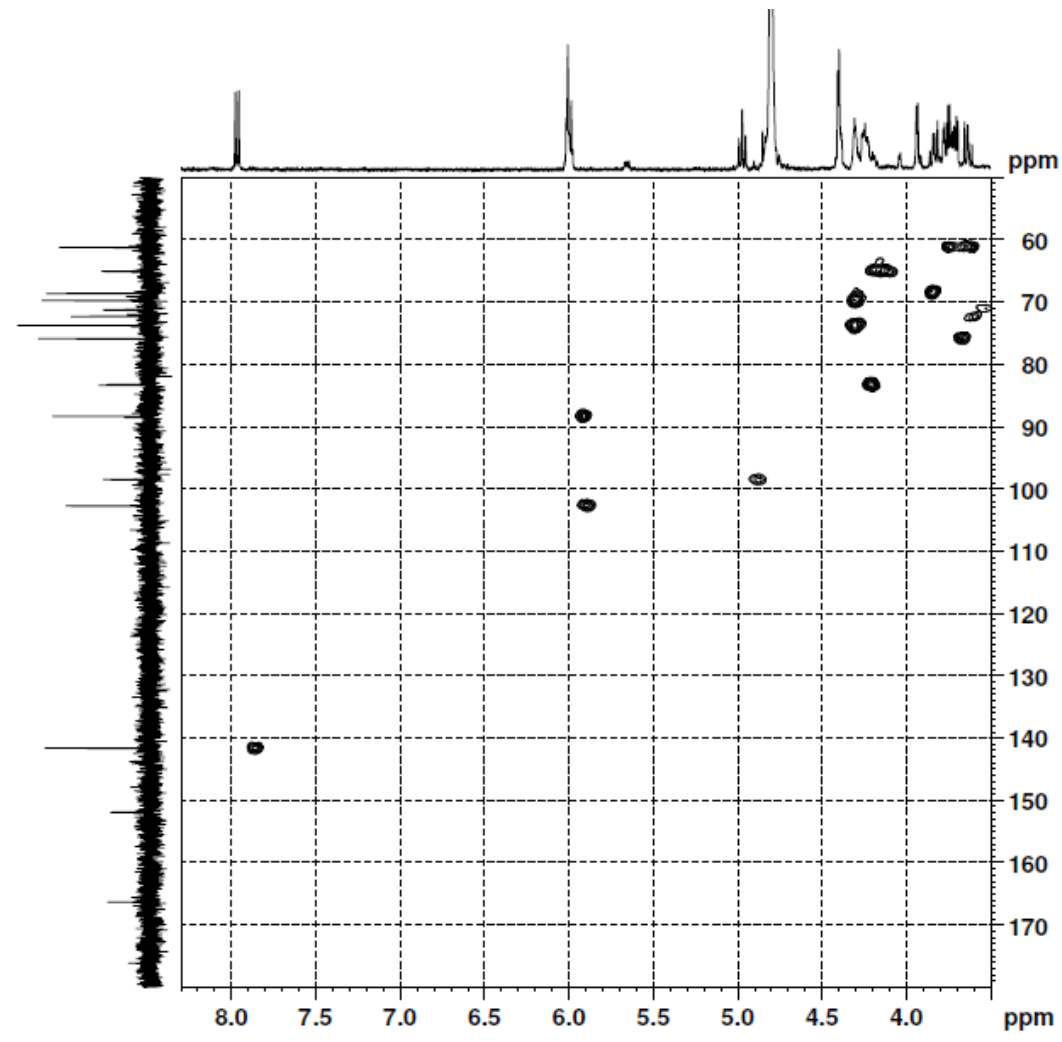

ESI-HRMS spectrum of UDP-galactose 3

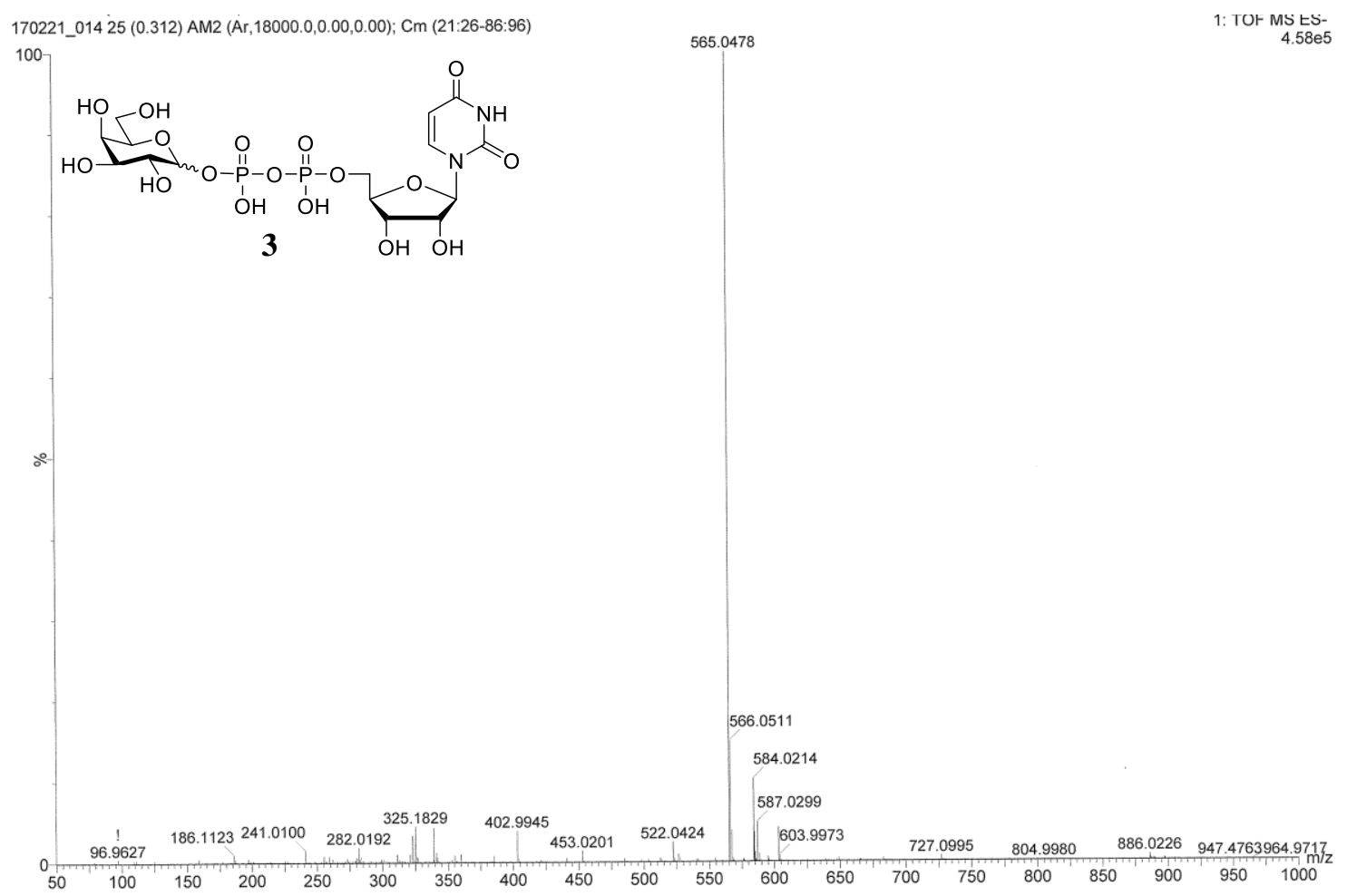


4) ${ }^{1} \mathrm{H},{ }^{13} \mathrm{C}$ and ${ }^{31} \mathrm{P}$ NMR, $,{ }^{1} \mathrm{H}^{-1} \mathrm{H} \mathrm{COSY},{ }^{1} \mathrm{H}_{-}{ }^{13} \mathrm{C}$ HMQC and ESI-MS spectra of UDP-mannose 4 ${ }^{1} \mathrm{H}$ NMR spectrum of UDP-mannose $4\left(400 \mathrm{MHz}, \mathrm{D}_{2} \mathrm{O}\right)$



${ }^{13} \mathrm{C}$ NMR spectrum of UDP-mannose $4\left(101 \mathrm{MHz}, \mathrm{D}_{2} \mathrm{O}\right)$<smiles>O=C1C2CCNCC1C2=O</smiles><smiles>O=c1ccn([C@@H]2O[C@H](COP(=O)(O)OP(=O)(O)OC3CC(O)CC(O)C3O)[C@H](O)C2O)c(=O)[nH]1</smiles>

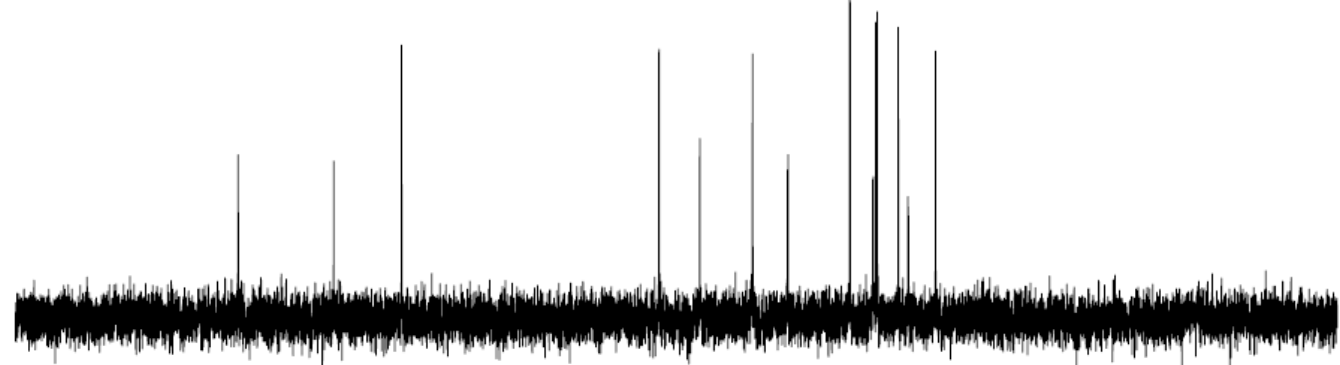

$\begin{array}{lllllllllllllllllll}190 & 180 & 170 & 160 & 150 & 140 & 130 & 120 & 110 & 100 & 90 & 80 & 70 & 60 & 50 & 40 & 30 & 20 & \mathrm{ppm}\end{array}$ 
${ }^{31} \mathrm{P}$ NMR spectrum of UDP-mannose $4\left(162 \mathrm{MHz}, \mathrm{D}_{2} \mathrm{O}\right)$
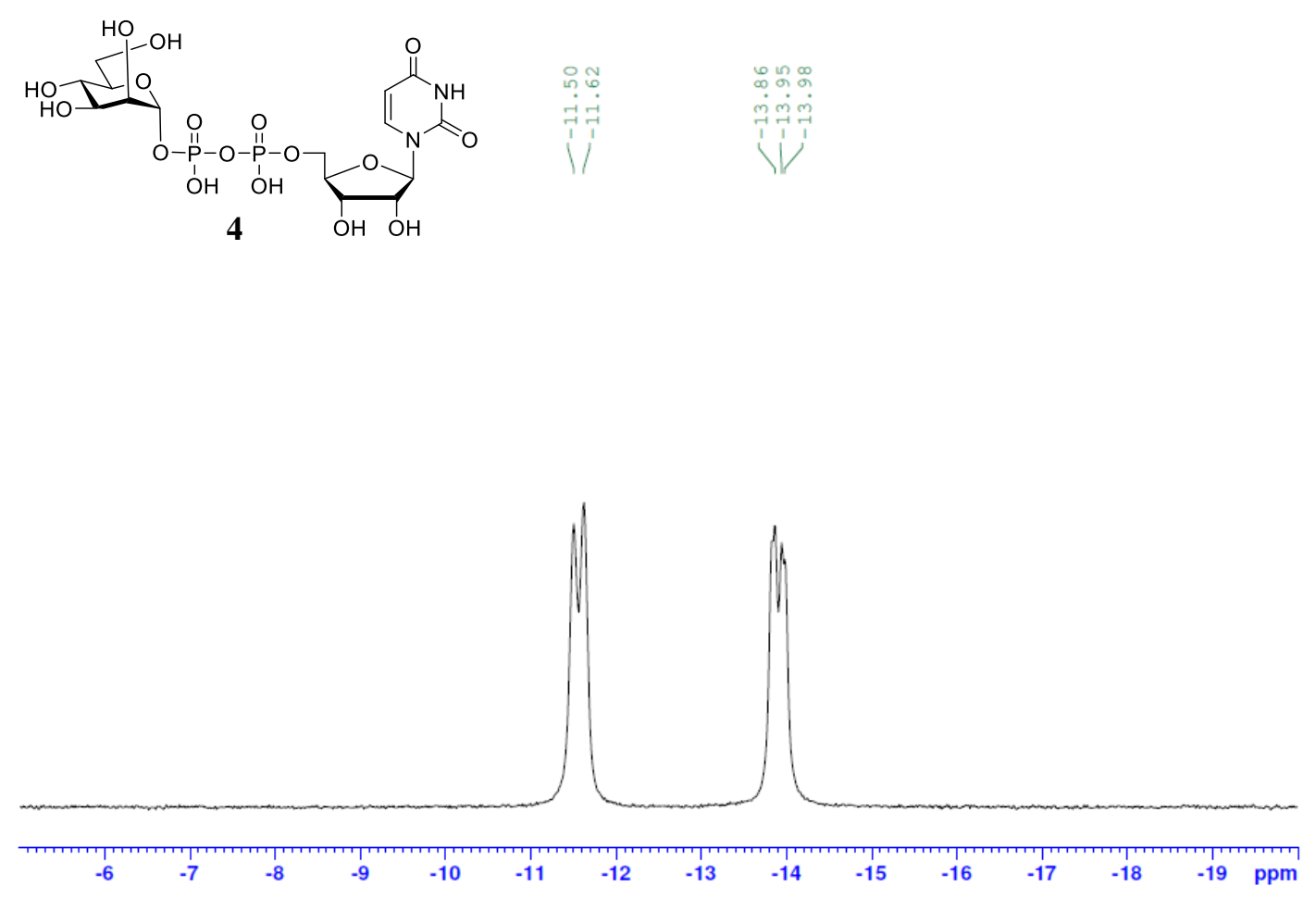

${ }^{1} \mathrm{H}-{ }^{1} \mathrm{H}$ COSY of UDP-mannose $4\left(\mathrm{D}_{2} \mathrm{O}\right)$

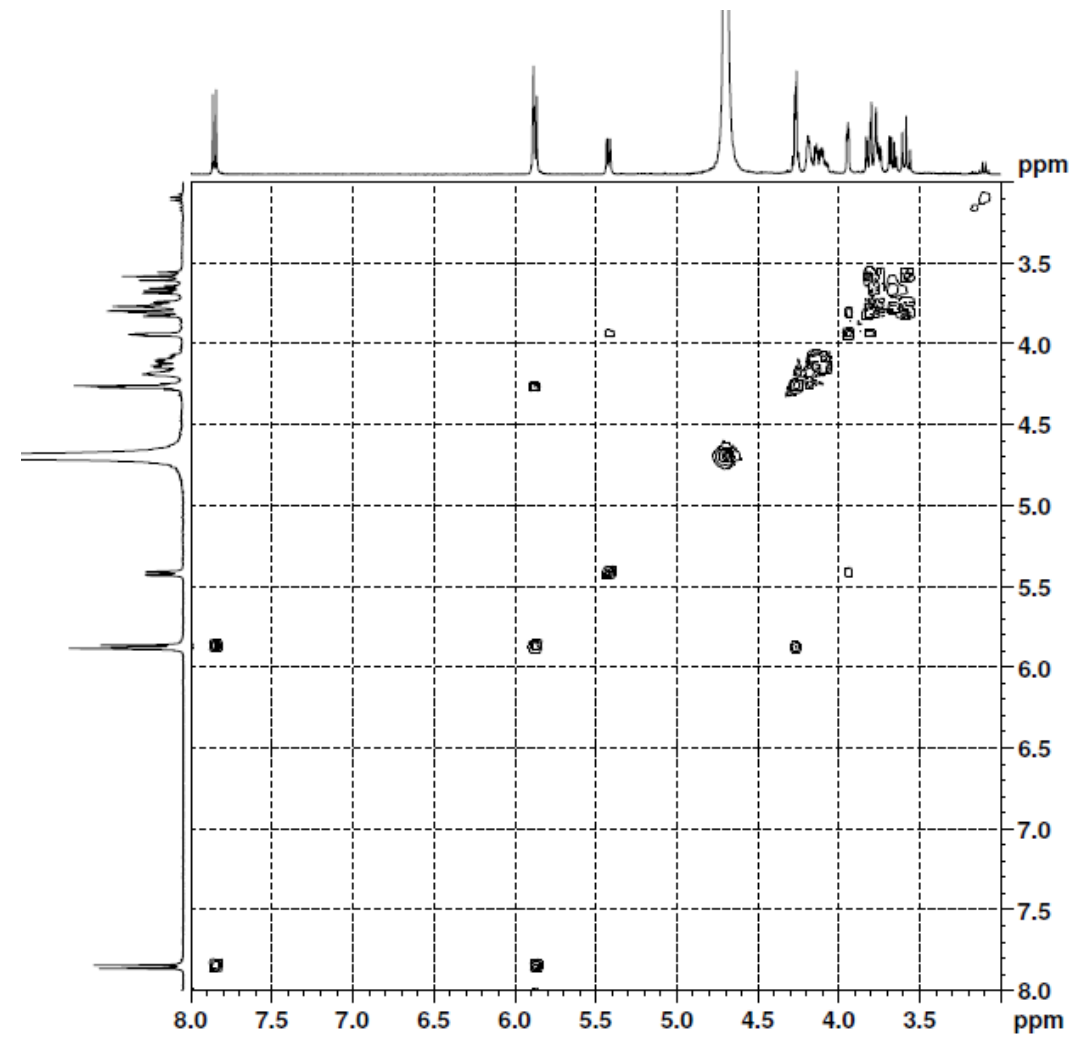


${ }^{1} \mathrm{H}-{ }^{13} \mathrm{C}$ HMQC of UDP-mannose $4\left(\mathrm{D}_{2} \mathrm{O}\right)$

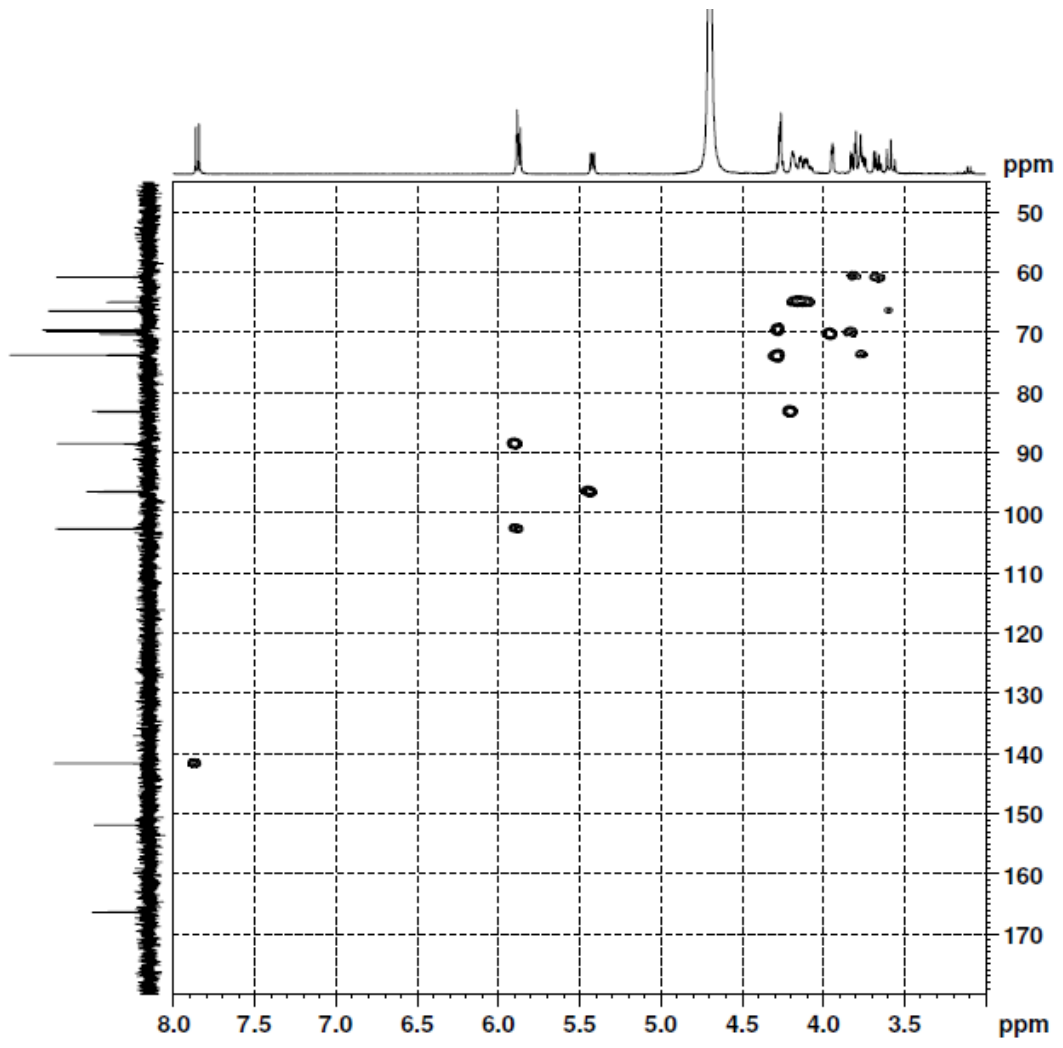

ESI-HRMS spectrum of UDP-mannose 4

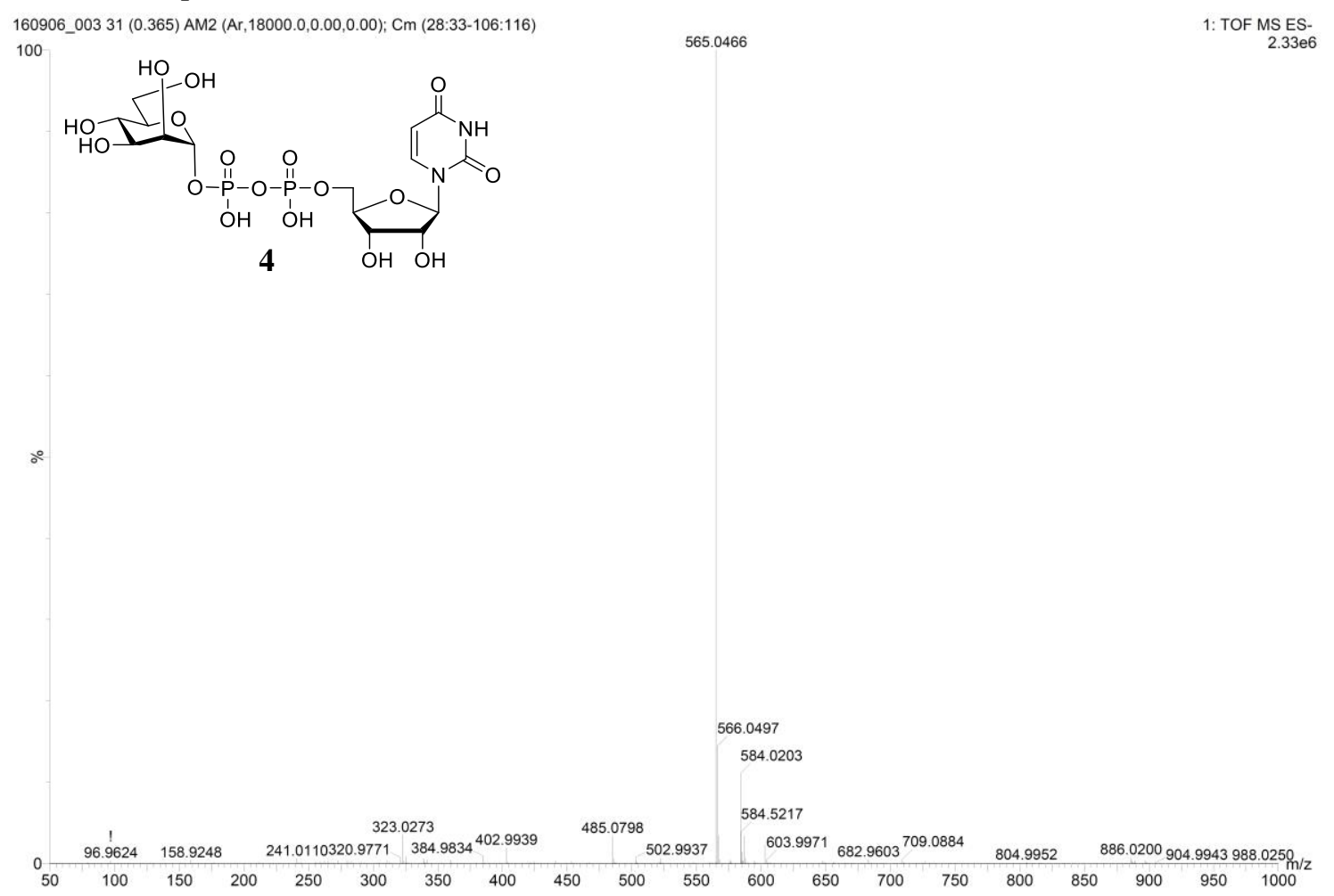


5) ${ }^{1} \mathrm{H},{ }^{13} \mathrm{C}$ and ${ }^{31} \mathrm{P}$ NMR,,${ }^{1} \mathrm{H}-{ }^{1} \mathrm{H}$ COSY,${ }^{1} \mathrm{H}_{-}{ }^{13} \mathrm{C}$ HMQC and ESI-MS spectra of GDP-mannose 5 ${ }^{1} \mathrm{H}$ NMR spectrum of GDP-mannose $5\left(400 \mathrm{MHz}, \mathrm{D}_{2} \mathrm{O}\right)$

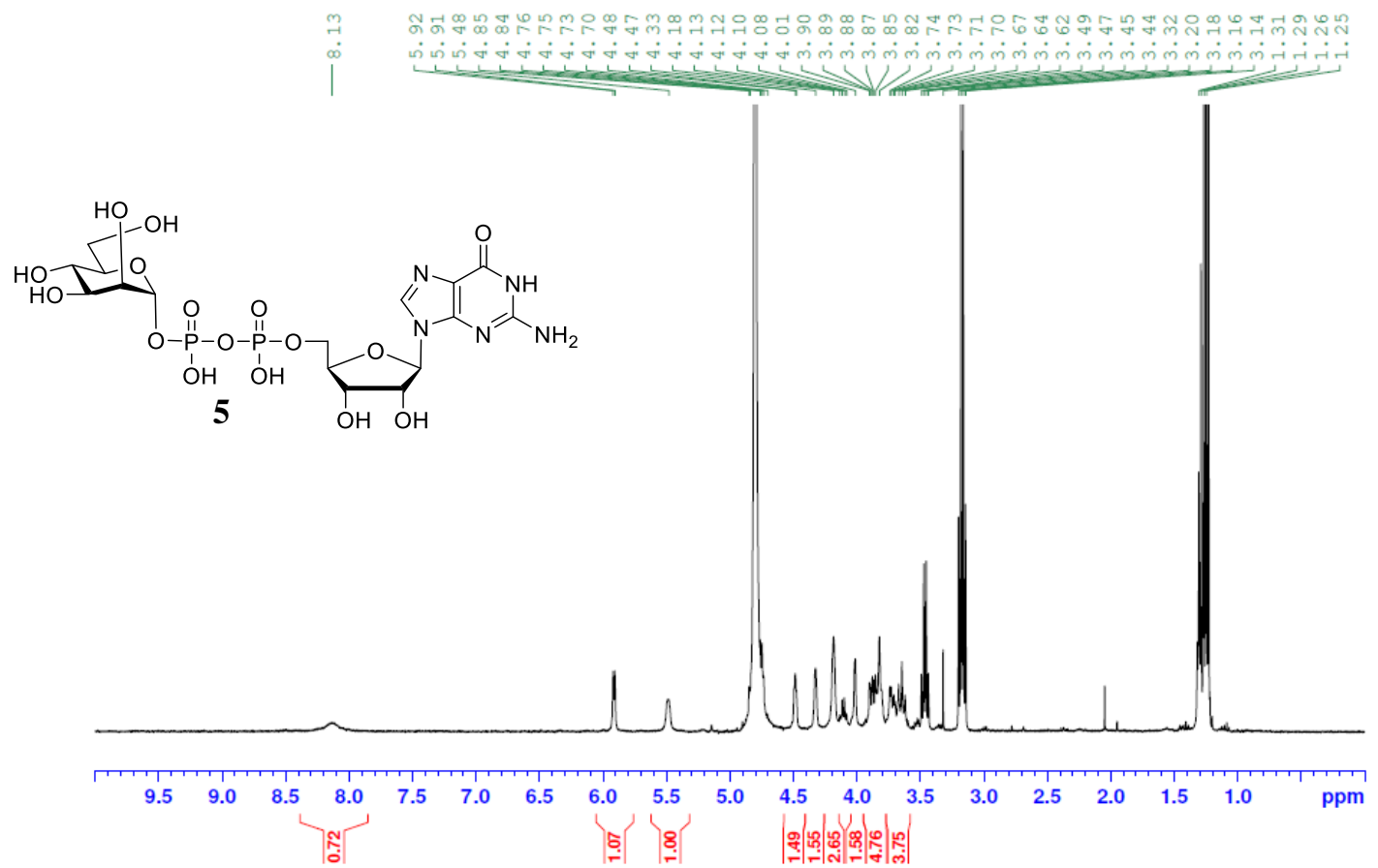

${ }^{13} \mathrm{C}$ NMR spectrum of GDP-mannose $5\left(101 \mathrm{MHz}, \mathrm{D}_{2} \mathrm{O}\right)$

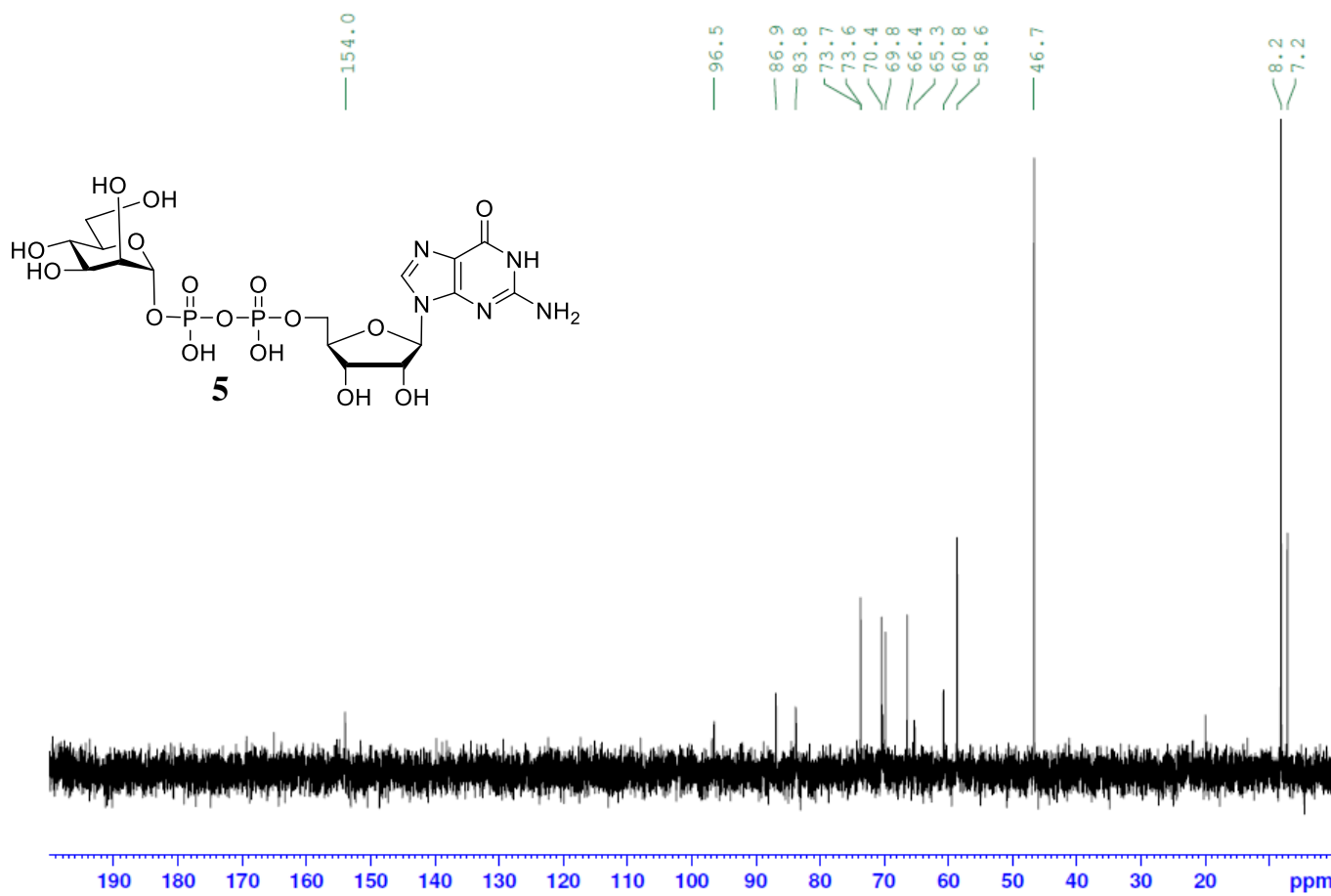


${ }^{31} \mathrm{P}$ NMR spectrum of GDP-mannose 5 (162 MHz, $\left.\mathrm{D}_{2} \mathrm{O}\right)$

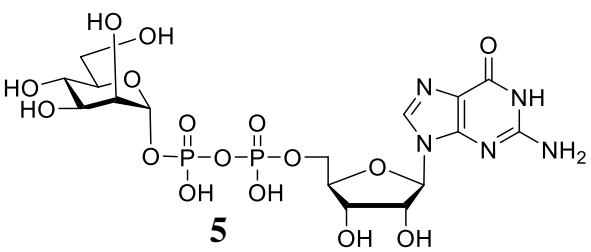

도유.

$\sqrt{1}$
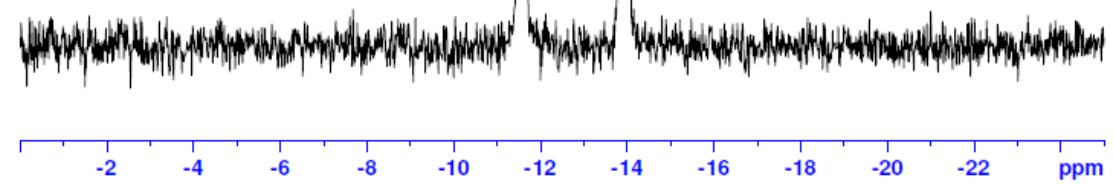

${ }^{1} \mathrm{H}-{ }^{1} \mathrm{H}$ COSY of GDP-mannose $5\left(\mathrm{D}_{2} \mathrm{O}\right)$

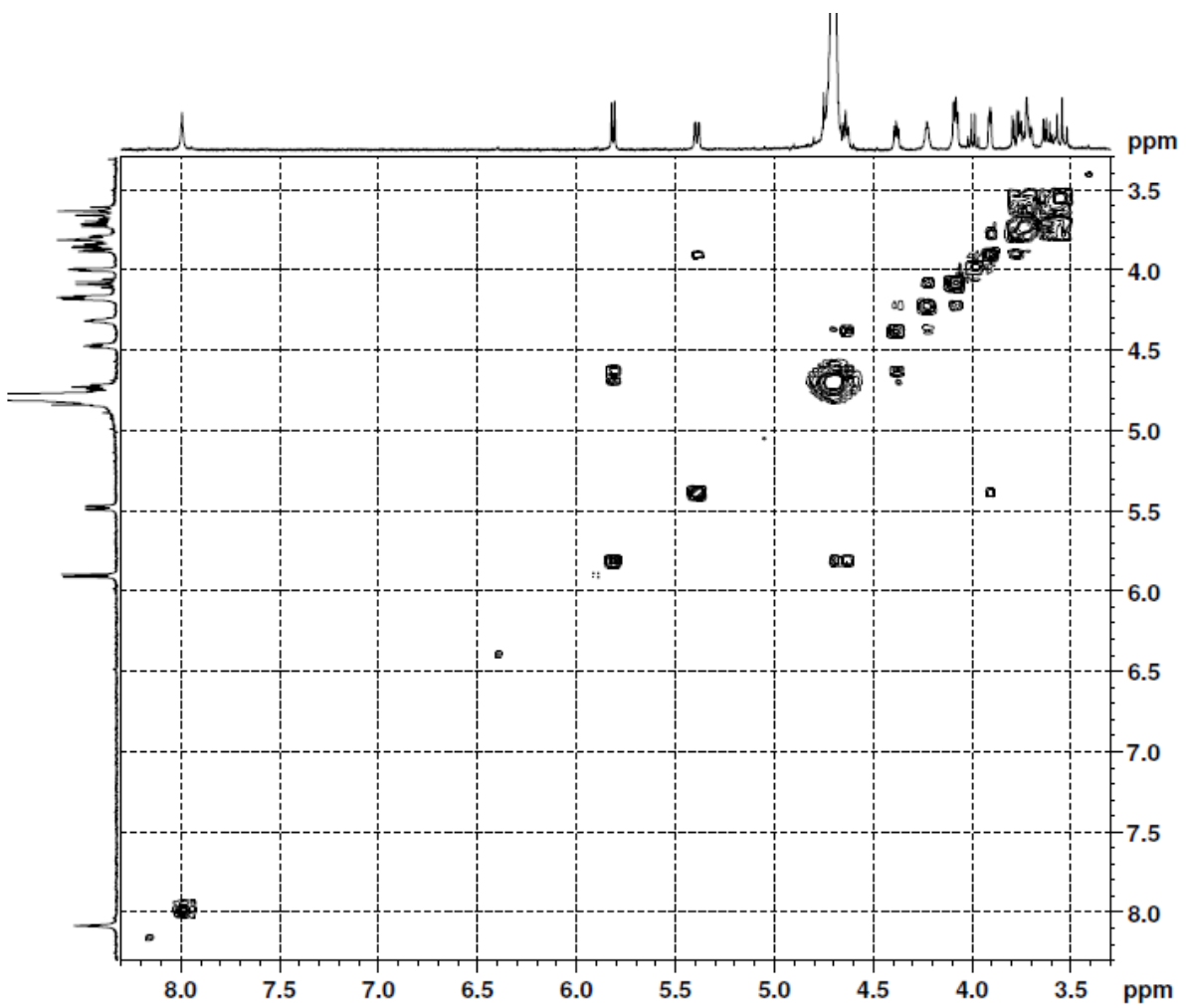


${ }^{1} \mathrm{H}-{ }^{13} \mathrm{C}$ HMQC of GDP-mannose $5\left(\mathrm{D}_{2} \mathrm{O}\right)$

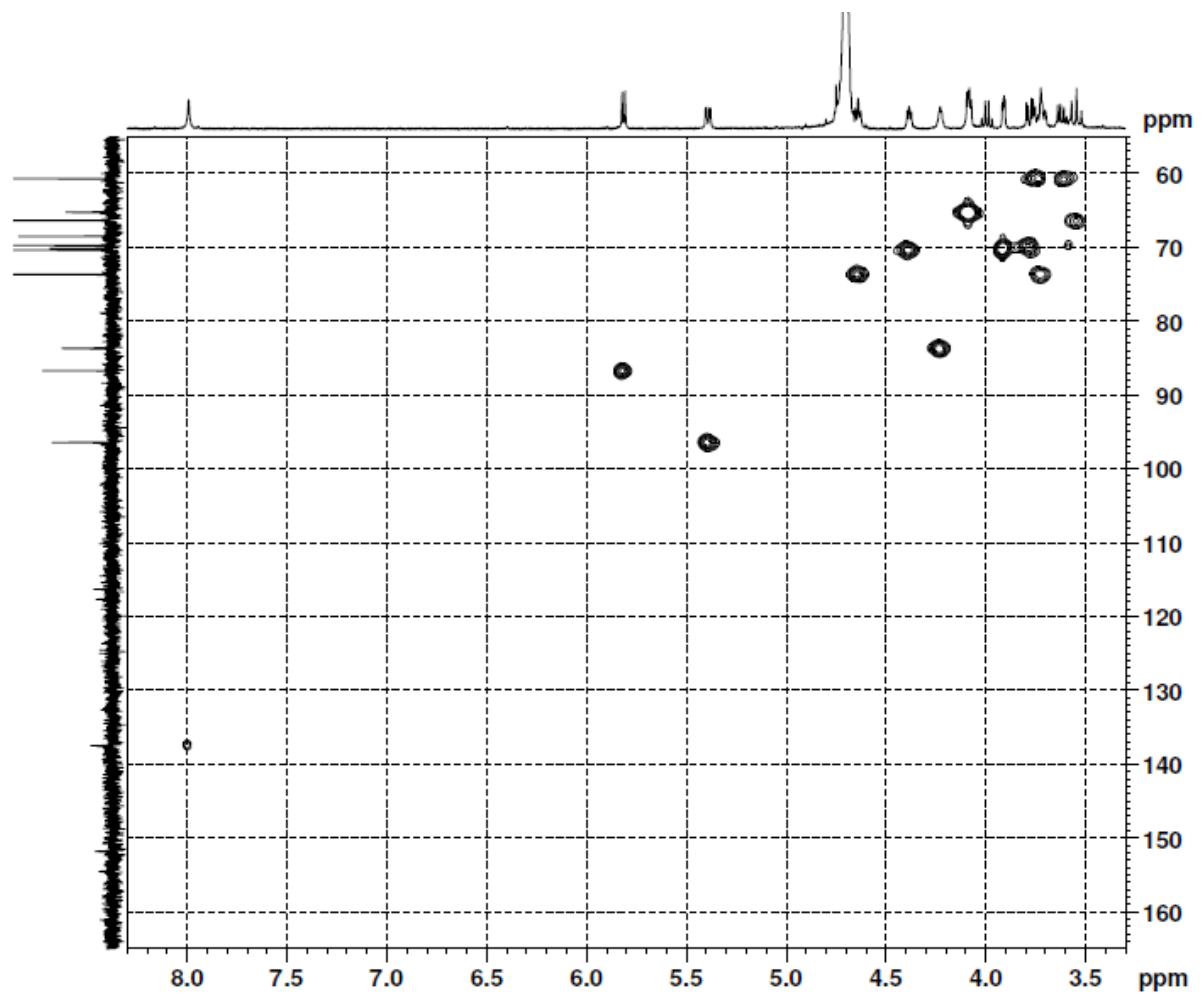

ESI-HRMS spectrum of GDP-mannose $\mathbf{5}$

160906_004 25 (0.312) AM2 (Ar, 18000.0,0.00,0.00); Cm (25:30-115:125) 100<smiles>NC1=Nc2c3ncn2C(OC2COP(=O)(O)OP(=O)(O)OC4C5CC(O)(OC4C(O)C2O)C(O)C(O)C5O)C(=N1)NC3=O</smiles>

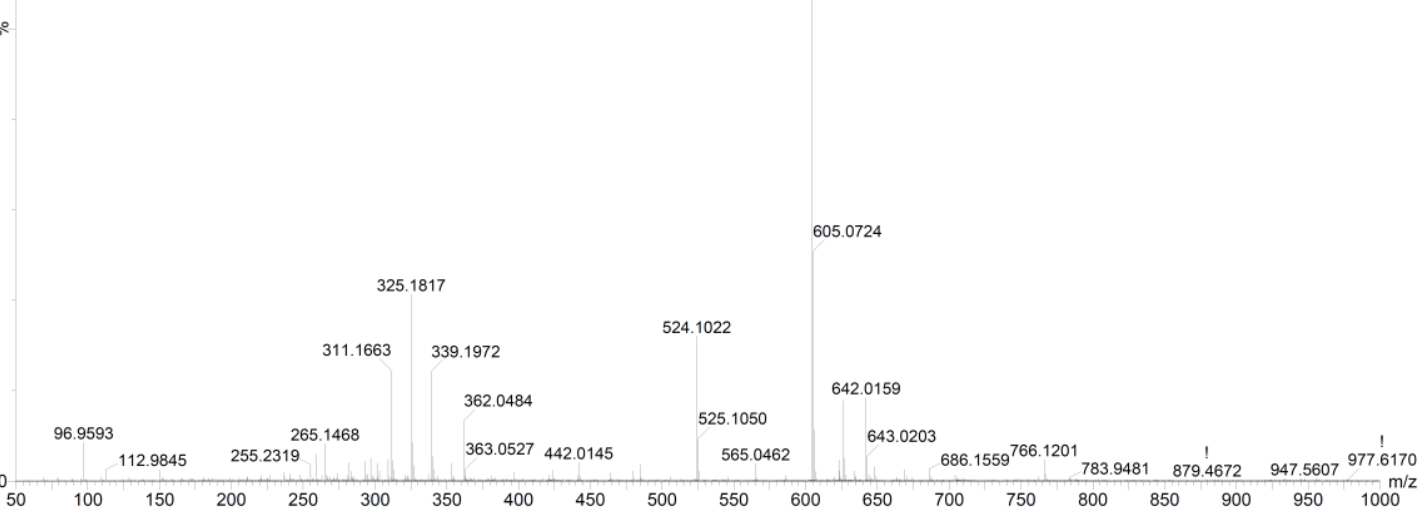

